\title{
Analyzing Multi-Channel Medium Access Control Schemes With ALOHA Reservation
}

\author{
Yunghsiang S. Han, Member, IEEE, Jing Deng, Member, IEEE, and Zygmunt J. Haas, Senior Member, IEEE
}

\begin{abstract}
In order to improve the throughput performance of Medium Access Control (MAC) schemes in wireless communication networks, some researchers proposed to divide a single shared channel into several sub-channels: one as control subchannel and the others as data sub-channels. In this paper, we analyze and evaluate the maximum achievable throughput of a class of generic multi-channel MAC schemes that are based on the RTS/CTS (Ready-To-Send/Clear-To-Send) dialogue and on ALOHA contention resolution. We study these multichannel MAC schemes under two split-channel scenarios: the fixed-total-bandwidth scenario and the fixed-channel-bandwidth scenario. In the fixed-total-bandwidth scenario, we show that the throughput of the multi-channel MAC schemes is inferior to that of the corresponding single-channel MAC scheme, which sends the RTS/CTS packets and DATA packets on a single shared channel. For the fixed-channel-bandwidth scenario, where CDMA or similar techniques can be applied, we derive the optimal number of the data sub-channels that maximizes the throughput. The analytical framework that we derive in this paper can also be used to evaluate other contention resolution technique, when the average contention period is known.
\end{abstract}

Index Terms - medium access control, MAC, shared channel, multiple channels, ALOHA, contention resolution, RTS/CTS dialogue

\section{INTRODUCTION}

In wireless communication networks, Medium Access Control (MAC) schemes are used to manage the access of active nodes to a shared channel [1]. As the throughput of the MAC schemes may significantly affect the overall performance of a wireless network, careful design of MAC schemes is necessary to ensure proper operation of a network. In order to reduce packet collisions and to improve throughput performance, some researchers proposed to divide the single shared channel into several sub-channels: one serves as a control sub-channel and the rest serve as data sub-channels. In this arrangement, the control sub-channel is used to reserve the data sub-channels, over which the data packets will be transmitted. Such reservations can be performed through the use of the RTS/CTS (Ready-To-Send/Clear-To-Send) dialogue [2]. Examples of multi-channel MAC schemes are [3]-[12].

Manuscript received July 2004; revised May 2005; accepted September 2005. The editor coordinating the review of this paper and approving it for publication is N. Mandayam. This work was supported in part by the SUPRIA program of the CASE Center at Syracuse University, by the National Science Council of Taiwan, R.O.C., under grants NSC 90-2213-E-260-007 and NSC 91-2213-E-260-021, and by grant number LABoR0078NR00C. Haas's work in this project was partially funded by the DoD Multidisciplinary University Research Initiative (MURI) programs administered by the Office of Naval Research under the grant number N00014-00-1-0564 and by the Air Force Office of Scientific Research under the grant number F49620-02-1-0233.

Corresponding author: Jing Deng, e-mail: jing.deng@ieee.org
Even though there are many multi-channel MAC schemes proposed in the technical literature, to the best of our knowledge, systematic comparison of these multi-channel MAC schemes with the corresponding single-channel schemes is not available except in [13]. However, a performance study of the multi-channel MAC schemes could provide invaluable insight into the MAC design methodologies, MAC parameters' settings, and how those affect performance such as the bandwidth efficiency of these schemes. Following the studies in [13], in this paper, we analyze the throughput performance of the multi-channel MAC schemes and compare them with the corresponding single shared channel MAC schemes.

In general terms, we analyze here a set of generic multichannel MAC schemes, which are assumed to be based on the RTS/CTS dialogue. ${ }^{1}$ In a contention resolution period, a ready node sends an RTS packet on the control sub-channel to reserve the use of a data sub-channel for future DATA packet transmission. The contention resolution technique for the control sub-channel is assumed to be pure ALOHA [14]. When the RTS packet is received, the intended receiver replies with a CTS packet to acknowledge the successful reservation of a data sub-channel, on which DATA packet transmission will commence soon. Further details regarding the scheme will be provided in the sequel.

Our analysis is based on a queueing model with $m$ servers and a maximum queue length of $q$. By modeling the successful RTS/CTS dialogue as a Poisson arrival, we derive the expected throughput of the multi-channel MAC schemes. We further consider two different scenarios for multiple-channel MAC schemes in wireless networks: the fixed-total-bandwidth scenario and the fixed-channel-bandwidth scenario. In the fixedtotal-bandwidth scenario, the total bandwidth being used by the network is fixed. Therefore, with more sub-channels, each sub-channel is allocated less bandwidth and, correspondingly, can carry lower data rate. In contrast, every sub-channel shares the same bandwidth in the fixed-channel-bandwidth scenario [8], but with more sub-channels, the network now requires more bandwidth. In practice, fixed-channel-bandwidth scenario can be realized, for example, by the CDMA technique.

In the fixed-total-bandwidth scenario, we determine the maximum achievable throughput of the multi-channel MAC scheme as a function of the number of the data sub-channels $m$, and of the ratio of the bandwidth of the control sub-

\footnotetext{
${ }^{1}$ Note that the purpose of the RTS/CTS dialogue is twofold: to reserve a data sub-channel for future data packet transmission and to solve the hidden/exposed terminal problems in multi-hop wireless networks, where at any time some neighbors can overhear either the sender or the receiver, but not both [2]. The focus of this paper is mainly in regards to the former issue.
} 
channel to that of the data sub-channel. We then compare these results to the throughput of the corresponding single-channel MAC scheme. We show that, when pure ALOHA technique is used for contention resolution of the access to the control sub-channel in a fully-connected network and when radio propagation delays are negligible, the throughput of the multichannel MAC scheme is inferior to that of the single-channel MAC scheme. In the fixed-channel-bandwidth scenario, we determine the maximum achievable throughput of the multichannel MAC scheme as a function of the number of the data sub-channels. Consequently, the optimal number of the data sub-channels that maximizes the throughput can be determined. Even though the proposed analysis is based on pure ALOHA contention resolution technique, the approach that we take in this paper can also be used with other contention resolution techniques, when average time of contention period is known or could be obtained.

For notational convenience, we term the single-channel MAC scheme as MAC-1 and the multi-channel MAC scheme as MAC-mD (where "D" represents "Data channels"). In the MAC-mD scheme, contention resolutions take place on the control sub-channel in parallel with the transmission of data packets on the data sub-channels.

The paper is organized as follows: in Section II, we overview related works. The assumptions and the notations used in this paper are summarized in Section III. In Sections IV and V, we present our analytical model and our main results on performance of the MAC-mD schemes. The numerical results and the simulation results are provided in Section VI. We summarize and conclude this work in Section VII, stating some possible future directions.

\section{RELATED WORK}

A split-channel MAC scheme was compared with a singlechannel MAC scheme in [15]. The authors categorized "scheduling epochs," the periods of time needed to schedule the next data transmission, into two groups: bandwidthdependent component (e.g., periods for contention resolution of reservation packets) and bandwidth-independent component (e.g., the propagation delay). It was found that, if a system has no bandwidth-independent component within its scheduling epochs, the split-channel schemes may achieve the same performance as the single-channel schemes. The analysis in [15] considered the average contention resolution period only, and their results may be inconclusive in general in regards to the advantage of the split-channel vs. the single-channel schemes, as reported by [13].

In [13], the performance of the dual channel MAC schemes were studied. The authors categorized a subset of MAC schemes, which use the RTS/CTS dialogue preceding the actual data transmission, into three groups: MAC-1, MAC2, and MAC-2R (cf. Fig. 1). ${ }^{2}$ In the MAC- 1 scheme, all the control packets and the data packets are transmitted on a single channel. In the MAC-2 and the MAC-2R schemes, however, the single shared channel is split into two sub-channels. One of these sub-channels, the control sub-channel, is used to transmit

\footnotetext{
${ }^{2} \mathrm{MAC}-2 \mathrm{R}$ is equivalent to the MAC-1D as defined in this paper.
}

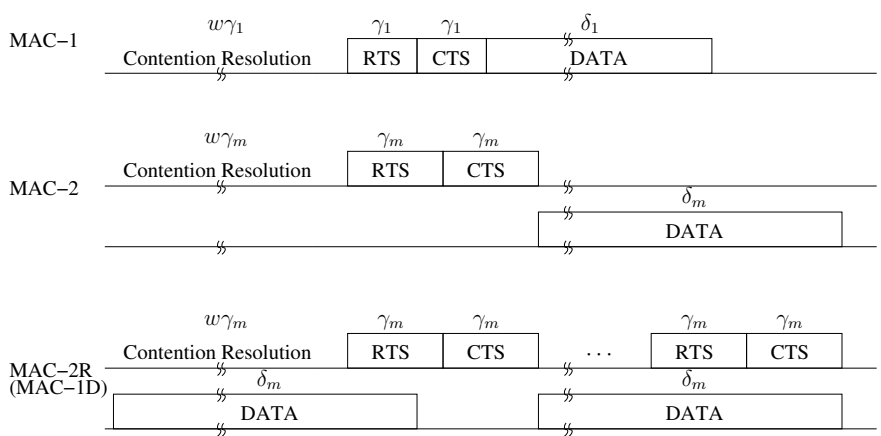

Fig. 1. Comparison of the MAC-1, MAC-2, and MAC-2R (MAC-1D) schemes

the RTS and the CTS control packets. The data packets are transmitted on the data sub-channel. The difference between the MAC-2 scheme and the MAC-2R scheme is that the MAC$2 \mathrm{R}$ scheme allows ready nodes to compete for and to reserve the use of the data sub-channel when the data sub-channel is currently being used. While it is obvious that the MAC$2 \mathrm{R}$ scheme out-performs the MAC-2 scheme, the performance comparison between the MAC-2R scheme and the MAC-1 scheme mandates further study. In [13], it has been proved that, when the ALOHA scheme is employed on the control sub-channel and propagation delays are negligible, the MAC1 scheme always out-performs the MAC-2R scheme. Our work is different from the work in [13], since our analysis applies to scenarios where $m$ can assume any value, not necessarily equal to 1.

A dynamic reservation technique for packet switching radio channels, called split-channel reservation multiple access (SRMA), was introduced in [3]. In SRMA, the available bandwidth is divided into three channels: two used to transmit control information and one used for the message transmission. Message delay of SRMA was studied in [3] and it was shown that SRMA out-performs other MAC schemes under some network settings. A queueing model was used to analyze the throughput of SRMA. However, as it is shown later, the model derived in our work is more accurate than the one given in [3]. Additionally, our model is more general, suitable for any number of data sub-channels.

Multiple-channel architectures were introduced as to overcome the deterioration in performance when a single very high-speed communication channel is used (due to the increasing ratio of propagation delay to data packet transmission time). For example, reference [4] proposes a multi-packet multi-channel CSMA/CD scheme to improve the throughput performance. Compared with previous work, in which a ready node can only compete for one channel out of multiple subchannels, the scheme in [4] allows nodes to compete at the same time for (randomly selected) multiple channels.

A multi-channel scheme was presented in [7] to improve the throughput of the IEEE 802.11 MAC scheme [16]. The scheme uses the Ad hoc Traffic Indication Messages (ATIM), which is transmitted by every node on a default channel to notify all neighboring nodes of the use of a certain data channel. By mandating contention resolution to be in the ATIM windows, 
only one transceiver is needed in every node. After an ATIM window period, the default channel can be used as a regular data channel to transmit data packets. This mechanism is an example of the fixed-channel-bandwidth MAC schemes.

Another multi-channel MAC scheme was proposed in [9]. The proposed scheme used Direct Sequence Spread Spectrum (DSSS) technique. In order to maintain the same subchannel data rate and the same frequency bandwidth usage, the processing gain of the spread spectrum signal is reduced when the number of sub-channels is increased. Transmission interference and packet error rate due to lower signal-to-noise ratio were studied with different processing gains. A waiting state, referred to as "pacing," was introduced to control the "pace" of transmissions at the control sub-channel. The pacing allows mitigation of the interference on the data sub-channels and reduction of the effect of near-far phenomenon. Our model in this paper differs from the model in [9] in the following aspects: a) the single shared channel is divided either in time domain or in frequency domain, and $b$ ) the processing gain of the spread-spectrum sub-channels is maintained at the same value. In our paper, we assume that packet collisions are the only source of packet loss on the wireless channel. Since the goal of our work is to study the throughput performance of multi-channel MAC schemes, we purposely exclude losses from sources other than packet collisions, such as transmission errors due to thermal noise or spectral interference. To include such other losses, the channel data rate in our study should be replaced by the data rate after an appropriate channel coding scheme is taken into account. ${ }^{3}$

\section{Assumptions And Notations}

In order to compare the throughput of the MAC- 1 and the MAC-mD schemes, we make here the following assumptions. For each of the schemes, we normalize the time, so that a "time unit" is the time to transmit a control packet (either RTS or CTS) in that scheme. Thus, the time to transmit a control packet is always 1 [time-unit]. The wireless communication networks that we study are assumed to be fully-connected; i.e., every node is in the transmission range of every other node in the network. We further assume that pure ALOHA contention resolution technique is used, that the overall traffic generated by active nodes (including retransmissions) is Poisson with aggregate arrival rate of $G$ [packets/time-unit], and that the packet processing and the radio propagation delays are negligible.

We establish the following notation:

- $L_{c}, L_{d}$ : the length (in bits) of a control (RTS/CTS) packet and that of a data packet, respectively

- $k$ : the ratio of data packet size to the control packet size; i.e., $k=\frac{L_{d}}{L_{c}}$

- $m$ : the number of data sub-channels

- $q$ : the size (in packets) of the virtual and distributed queue used in the MAC-mD scheme

- $R, R_{c}$, and $R_{d}$ : the data rates (in bits-per-second) of the entire shared channel, the control sub-channel, and the data sub-channel, respectively; i.e., $R=R_{c}+m R_{d}$

\footnotetext{
${ }^{3}$ In other words, we assume that the channel data rate is the actual data rate after channel coding, rather than the raw channel data rate.
}

- $r$ : the ratio of the data rate of the control sub-channel to the data rate of the data sub-channel channel in the MAC-mD scheme; i.e., $r=\frac{R_{c}}{R_{d}}$

- $\gamma_{1}, \delta_{1}$ : the transmission time (in seconds) of a control packet and the transmission time (in seconds) of a data packet, respectively, in the MAC-1 scheme; i.e., $\gamma_{1}=\frac{L_{c}}{R}$ and $\delta_{1}=\frac{L_{d}}{R}=k \gamma_{1}$

- $\gamma_{m}, \delta_{m}$ : the transmission time (in seconds) of a control packet and the transmission time (in seconds) of a data packet, respectively, in the MAC-mD scheme; i.e., $\gamma_{m}=$ $\frac{L_{c}}{R_{c}}=\gamma_{1} \frac{r+m}{r}$ and $\delta_{m}=\frac{L_{d}}{R_{d}}=k \gamma_{1}(r+m)$

- $\delta$ : normalized data packet transmission time (in timeunits) in the MAC-mD scheme; i.e., $\delta=\frac{\delta_{m}}{\gamma_{m}}=k r$.

Note that, in the fixed-channel-bandwidth scenario, $r=1$.

\section{THE MAC-MD SCHEME}

In what follows, we describe the mechanism of the MAC-mD scheme (an example of MAC-1D is shown in Fig. 1$)^{4}$

1) We use the notion of a virtual and distributed queue, which is an imaginary queue that "stores" all the data packets arriving at the network nodes. It is assumed that the status of the virtual queue is precisely known to all network nodes. Further discussion on the distributed queue is provided in the Appendix.

2) Every node with data packets to send can compete on the control sub-channel by sending RTS packet to its intended receiver, which should reply with a CTS packet based on the operational rules of the RTS/CTS dialogue [2].

3) When the CTS packet is received at the sender of the RTS packet, the sender/receiver pair becomes the "winner" of the current competition (i.e., current contention).

4) The following are the rules that a winner of a competition follows:

a) If there is at least one available (idle) data subchannel after the prior requests in the distributed queue are assigned data sub-channels, the winner of a competition transmits on an available data sub-channel immediately after the CTS packet is received.

b) If all data sub-channels are busy and the distributed queue is not full, the winner of the competition adds itself to the distributed queue and waits for an available data sub-channel.

c) If all data sub-channels are busy and the distributed queue is full, the winner of the competition drops its right to transmit and returns to compete on the control sub-channel. ${ }^{5}$

\footnotetext{
${ }^{4}$ The results of the MAC-1 throughput can be found in [13]; we use these results here to compare the throughput of the MAC-mD and the MAC-1 schemes.

${ }^{5}$ In practical wireless networks, however, network nodes should stop competing on the control sub-channel when they know that winners of a competition will have to drop their rights to transmit. The presented contention mechanism allows us to develop a very simple and tractable analytical model that is closest to the real situation.
} 


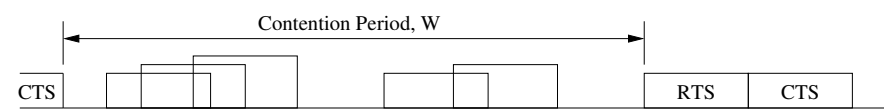

Fig. 2. Illustration of Contention Periods, $W$

5) After transmission on a data sub-channel, the sender returns to compete on the control sub-channel for the right of transmission for its next data packet.

6) Whenever a data sub-channel becomes available due to the conclusion of its current data transmission, it removes and serves one of the customers in the distributed queue. If the queue is empty, the data subchannel becomes idle.

Since in our model, the network is fully connected, every reservation and every transmission are overheard by all the network nodes. In our queueing model, the virtual and distributed queue is used to represent the information of backlogged data packets waiting for transmission. The virtual queue is served by $m$ servers (i.e., the $m$ data sub-channels) and can store at most $q$ data packets. A new winning transmission request joins the virtual queue if there are $m$ other customers in the system currently being served. Whenever the service of any of the currently $m$ customers is completed, the first customer waiting in the distributed queue enters the now-idle server; i.e., will be assigned the corresponding data sub-channel.

In the following discussion we use the $M / D / 1 / 1+q$ and $M / M / m / m+q$ model to analyze the throughput of the MAC-mD scheme.

\section{Performance Analysis of the MAC-mD Schemes BY QUEUEING MODEL}

In order to apply the above queueing model to analyze the MAC-mD family of schemes, we specify the arrival statistics and the service statistics of the system.

\section{A. Arrival Statistics}

Even though the behavior of the contention resolution process on the control sub-channel is quite complicated, completion times of successful RTS/CTS dialogues have been successfully modeled in the past by a Poisson process [3]. In order to present a tractable analytical result, we borrow this approach, modeling the completion times of successful RTS/CTS dialogues on the control sub-channel as a Poisson process with rate of $\lambda$ [arrivals/time-unit]. We will further justify this choice by our simulation results in Section VI-B.

The control sub-channel is basically an ALOHA channel with every successful RTS packet leading to a CTS packet transmission. Let the aggregate arrival rate of data traffic at active nodes be $G$ [packets/ time-unit]. The duration of the contention period, shown in Fig. 2, after the control subchannel becomes available for a new competition and before the new competition is completed (i.e., a successful RTS packet is transmitted and received correctly) can be calculated as ( [13]):

$$
W=\frac{1}{G e^{-2 G}}-1,
$$

in units of $\gamma_{m}$.

The average completion rate of successful RTS/CTS dialogues in a time-unit can be calculated as:

$$
\lambda=\frac{1}{W+2}=\frac{G e^{-2 G}}{1+G e^{-2 G}} .
$$

In the steady state, the throughput of the whole system is at most the capacity of the control sub-channel. Therefore, in this analysis, we are only interested in the largest value of $\lambda$, which is $\lambda=0.1554$, when $G=0.5$.

For any contention resolution scheme other than the ALOHA scheme presented here, the value of $\lambda$ can be obtained if the average time of contention period is known. Therefore, our analysis could be used in any scheme in which the average time of the contention periods is known.

The analysis that follows is based on the assumption of Poisson arrivals of the transmission requests. Therefore, strictly speaking, our results do not hold in systems in which the Poisson arrival assumption is violated. However, as suggested by the simulation results in Section VI-B, our results provide a close approximation of a real network, when the number of competing nodes is relatively large.

\section{B. Service Statistics}

When the data packet length is $L_{d}$, the service time for the customer in the queue is $\delta$. Since the service time is a constant, the queueing model to analyze the MAC-mD scheme should have a determinate service time. In the following queueing model, $M / D / 1 / 1+q$ model with only one server (data subchannel) will be used to analyze the MAC-1D scheme. Due to the lack of the suitable queueing model when $m>1$, $M / M / m / m+q$ model will be used for $m>1$. Therefore, we assume that the lengths of data packets are the same when $m=1$ and that the lengths of data packets are exponentially distributed with an average of $L_{d}$ for $m>1$. In Section VI$\mathrm{B}$, we show that the performance of the fixed-packet-length scenario is a very close approximation of our analytical results with exponentially distributed packet lengths.

\section{Throughput of MAC-1D}

In this subsection, we study the throughput of the MAC-1D scheme, which is equivalent to the MAC-2R scheme in [13].

Let $\alpha_{i}$ be the probability that $i$ customers (after successful RTS/CTS dialogues on the control sub-channel) arrive to the system during the service time $\delta$. According to the Poisson arrival assumption, we have

$$
\alpha_{i}=\frac{(\lambda \delta)^{i}}{i !} e^{-\lambda \delta}=\frac{\rho^{i}}{i !} e^{-\rho} \quad i=0,1, \cdots,
$$

where $\rho=\lambda \delta=\lambda k r$ is the utilization factor.

Let $p_{n}^{(d)}, n=0,1, \ldots, q$, be the steady state probabilities of the virtual queue occupancy at the departure instants of customers from the system. These probabilities can be obtained by solving the following recursive equations in the normalized variable $p_{n}^{(d)} / p_{0}^{(d)}([17])$ :

$$
\frac{p_{n+1}^{(d)}}{p_{0}^{(d)}}=\frac{1}{\alpha_{0}}\left(\frac{p_{n}^{(d)}}{p_{0}^{(d)}}-\sum_{j=1}^{n} \frac{p_{j}^{(d)}}{p_{0}^{(d)}} \alpha_{n-j+1}-\alpha_{n}\right)
$$


for $n=0,1, \ldots, q-1$ and then solving for $p_{0}^{(d)}$ by using

$$
p_{0}^{(d)}=\frac{1}{\sum_{n=0}^{q} \frac{p_{n}^{(d)}}{p_{0}^{(d)}}},
$$

which is equivalent to $\sum_{n=0}^{q} p_{n}^{(d)}=1$.

Note that the above steady state probabilities are evaluated at the instants of customers' departures. We are interested in the steady-state probabilities, $\pi_{n}$, that there are $n$ customers in the system at any time instant, $n=0,1, \ldots, q, q+1$. Let $p_{n}^{(a)}, n=0,1, \ldots, q, q+1$, be the steady-state probability that there are $n$ customers in the system, as seen by a customer arriving to the system. By the PASTA property [17], we have that

$$
\pi_{n}=p_{n}^{(a)} \quad n=0,1, \ldots, q, q+1 .
$$

Let $p_{n}^{(a c)}, n=0,1, \ldots, q$, be the steady-state probabilities that there are $n$ customers in the system, as seen by an arriving customer that actually enters the queue. Due to the fact that the state of the system can only change by at most \pm 1 between two time instants and by Kleinrock's result [18] we have

$$
p_{n}^{(d)}=p_{n}^{(a c)} n=0,1, \ldots, q .
$$

Since the blocking probability is $\pi_{q+1}$, we obtain that

$$
\pi_{n}=\left(1-\pi_{q+1}\right) p_{n}^{(d)} \quad n=0,1, \ldots, q .
$$

These probabilities can be calculated using the following equations:

$$
\pi_{n}=\left\{\begin{array}{ll}
\frac{1}{p_{0}^{(d)}+\rho} p_{n}^{(d)} & n=0,1, \ldots, q \\
1-\frac{1}{p_{0}^{(d)}+\rho} & n=q+1
\end{array},\right.
$$

and,

$$
\sum_{n=0}^{q+1} \pi_{n}=1
$$

The throughput can now be computed as follows:

$$
S_{m}(\mathrm{MAC}-1 \mathrm{D})=\frac{1}{r+1} \cdot \frac{\rho}{p_{0}^{(d)}+\rho},
$$

where we have used (1), (2), and $m=1$.

Since any arrival is dropped when the queue is full (i.e., when the system is in the state $q+1)$, the average arrival rate of customers who actually enter the system is $\lambda_{e}=\lambda\left(1-\pi_{q+1}\right)$. Therefore, the effective utilization factor is:

$$
\rho_{e}=\rho\left(1-\pi_{q+1}\right) .
$$

The system will be stable when the effective utilization factor is less than 1; i.e., when

$$
\rho_{e}=\lambda k r\left(1-\pi_{q+1}\right)<1 .
$$

Hence, to determine the maximum achievable throughput, we need to maximize (3) under the constraint that $\rho_{e}<1$. When $m=1$ and $q=1$, the throughput is maximized when $p_{0}^{(d)}=$ $e^{-\rho}$. Hence, the throughput of a MAC-1D scheme with $q=1$ is

$$
S_{m}(\mathrm{MAC}-1 \mathrm{D})=\frac{\rho}{(1+r)\left(\rho+e^{-\rho}\right)} .
$$

The maximum value of (4) can be obtained by optimizing $r>0$. Since the effective utilization factor of the queue is $\frac{\rho}{\rho+e^{-\rho}}<1$, such throughput is the achievable (stable) maximum throughput of a MAC-1D scheme with $q=1$. In Section VI-A.1, we will compare the maximum throughput obtained by maximizing (3) to that given in [13].

\section{Throughput of MAC- $m D(m>1)$}

With more than one data sub-channel, we apply the $M / M / m / m+q$ model to analyze the throughput, where we assume that the service time is exponentially distributed with average of $1 / \mu=\delta$. Our simulation results in Section VI-B show that the behavior of the MAC-mD schemes with fixed data packet length is approximated well by the $M / M / m / m+$ $q$ queueing model with parameters $\mu, \lambda, m$ and $q$.

For the $M / M / m / m+q$ model, the steady-state probabilities are given as ( [19]):

$$
\pi_{n}=\left\{\begin{array}{ll}
\pi_{0} \frac{(m \rho)^{n}}{n !}, & n \leq m \\
\pi_{0} \frac{m^{m} \rho^{n}}{m !}, & m<n \leq m+q
\end{array},\right.
$$

where $\rho=\frac{\lambda}{m \mu}$ is the utilization factor.

Using the condition $\sum_{n=0}^{m+q} \pi_{n}=1$, we calculate $\pi_{0}$ to be:

$$
\pi_{0}=\frac{1}{\sum_{n=0}^{m-1} \frac{(m \rho)^{n}}{n !}+\sum_{n=m}^{m+q} \frac{(m \rho)^{n}}{m !} \frac{1}{m^{n-m}} .}
$$

The throughput is then:

$$
\begin{aligned}
S_{m} & =\frac{1}{R} \cdot R_{d}\left(\sum_{n=1}^{m+q} \min \{n, m\} \cdot \pi_{n}\right) \\
& =\frac{1}{r+m}\left(\sum_{n=1}^{m+q} \min \{n, m\} \cdot \pi_{n}\right) .
\end{aligned}
$$

The maximum achievable throughput can be obtained by maximizing (5) under the constraint that the system is stable, meaning that the effective utilization factor is less than 1 :

$$
\rho_{e}=\rho\left(1-\pi_{m+q}\right)<1 .
$$

When $m$ is large enough such that $\pi_{m+q} \ll 1, \rho_{e}$ is close to $\rho$. By setting $\rho_{e} \approx \rho \approx 1$, the maximum throughput of the MAC-mD schemes is achieved. That is,

$$
\rho=\frac{\lambda}{m \mu}=\frac{\lambda k r}{m} \approx 1,
$$

meaning that

$$
r=r^{*} \approx \frac{m}{\lambda k}
$$

maximizes the throughput of the MAC-mD schemes. These results will be further substantiated in Section VI. 


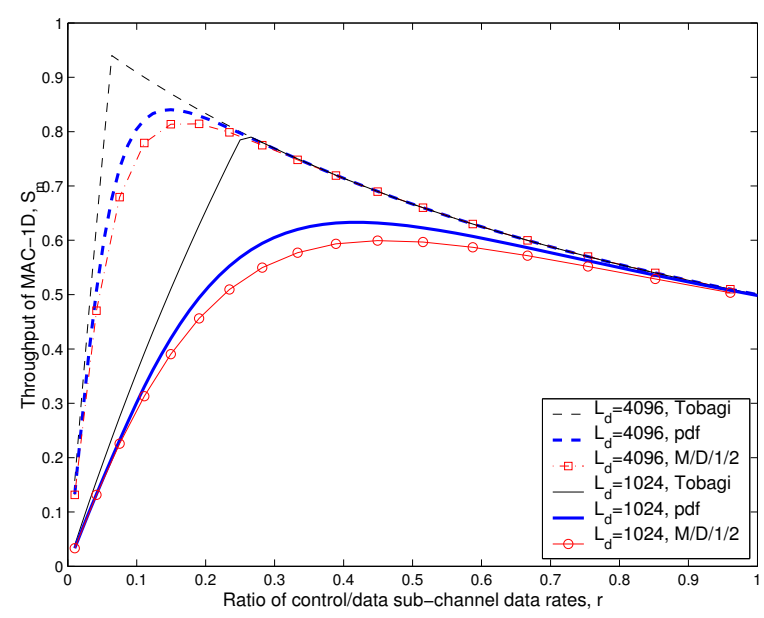

Fig. 3. Throughput of the MAC-1D scheme for $q=1$ and for the fixedtotal-bandwidth scenario

\section{NUmericAl AND Simulation Results}

In this section, we present the numerical and simulation results, where the fixed-total-bandwidth scenario is considered, except for those cases where indicated otherwise. The control packet length is fixed at 48 bits in these studies.

\section{A. Numerical Results}

1) The MAC-1D Scheme: We present our M/D/1/2 numerical results in Fig. 3. We also show the numerical results from [13], which considered the Probability Density Function (pdf) of the arrival time of successful RTS/CTS dialogues. The two sets of results match quite well. In order to compare our proposed analysis with the maximum achievable throughput $C_{R M}$ for a given $r$ presented in [3], ${ }^{6}$ we provide $C_{R M}$ in terms of our notations as follows:

$$
C_{R M}=\min \left(\frac{k r}{2 e(1+r)}, \frac{1}{1+r}\right)
$$

and we plot it in Fig. 3. It can be observed that the results of our analysis proposed in this paper and based on the assumption of Poisson arrival of successful RTS/CTS dialogues are quite close to the results presented in [13] (without such a Poisson assumption). Therefore, (4) predicts a reasonably good closed-form approximated throughput for the MAC schemes studied in [13]. In Fig. 3, we can observe that the maximum achievable throughput of the proposed analytical model is more accurate than that given in [3]. Compared with [3], our results of optimum $r$, which maximizes the throughput of the MAC-1D scheme, are almost identical to the more accurate results in [13].

Figure 4 studies the effect of different queue sizes on the throughput of the MAC-1D scheme. The data packet length is fixed at $L_{d}=1024$. When $q=0$ (i.e., no reservation is allowed in the MAC-1D scheme), its throughput is significantly lower than those schemes with positive queue size. This can be explained as follows: since no reservation is allowed, one subchannel (e.g., the control sub-channel) is being wasted while

\footnotetext{
${ }^{6}$ This result has been converted to the case of one control sub-channel and one data sub-channel.
}

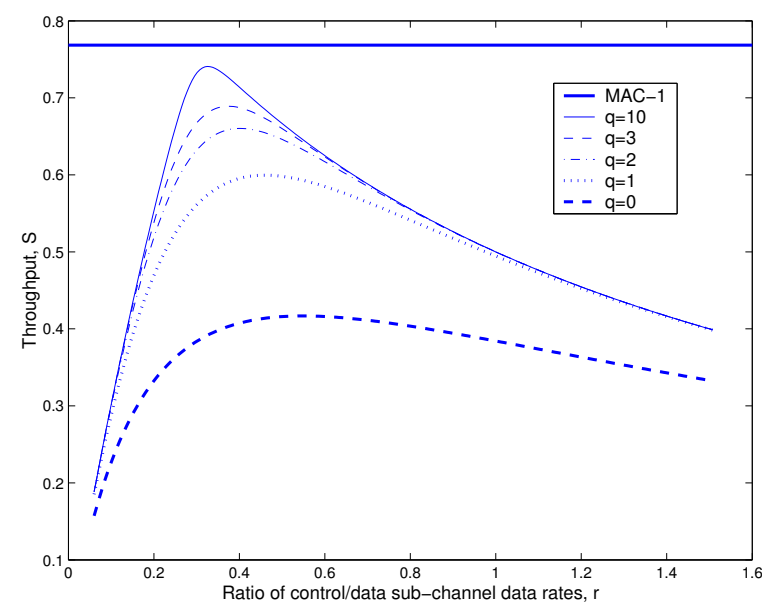

Fig. 4. Throughput of the MAC-1D scheme for $L_{d}=1024$ and for the fixed-total-bandwidth scenario

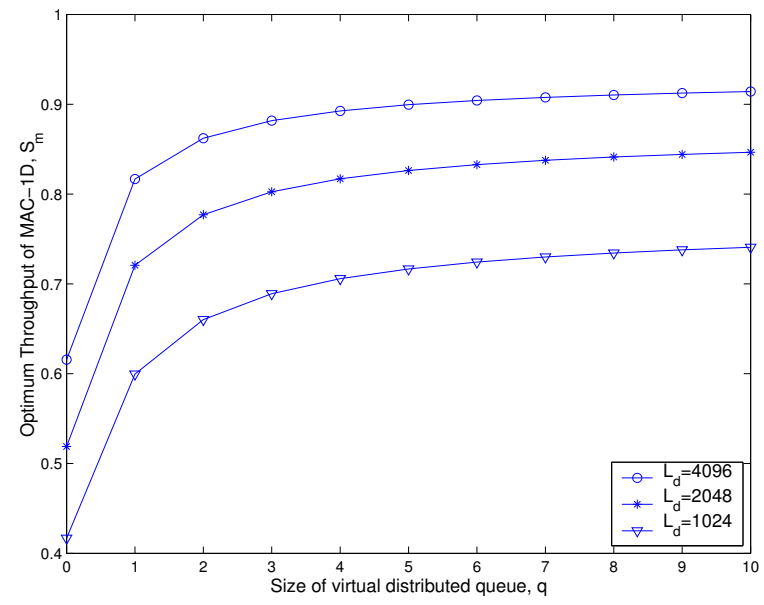

Fig. 5. Optimal throughput of the MAC-1D scheme for the fixed-totalbandwidth scenario

the other sub-channel (e.g., the data sub-channel) is being used to transmit packets. Channel contention can only restart after the current data transmission is finished (similar to the MAC-2 scheme in [13]). As expected, the throughput performance of MAC-1D scheme is better with larger queue size. Optimum values of $r$ can be found to maximize the throughput of the MAC schemes. Interestingly, when the MAC-1D schemes operate away from the optimum values of $r$ (with either too small or too large values of $r$ ), the performance of different queue sizes is almost identical. This is due to the fact that the reservation queue is always empty/full when $r$ is too small/large. When the queue is always empty, the data subchannels are more likely to be idle. When the queue is always full, the control sub-channel is too large and is wasting the bandwidth. In both cases, the actual size of the queue $(q>0)$ hardly affects the throughput performance. We also include the throughput of the MAC-1 scheme in Fig. 4. The optimum throughput of the MAC-1D scheme approaches that of the MAC-1 scheme as $q$ increases.

In Fig. 5, we show the effect of $q$, the size of the virtual and distributed queue, on the optimum throughput performance for different data packet length. The throughput shown in this 


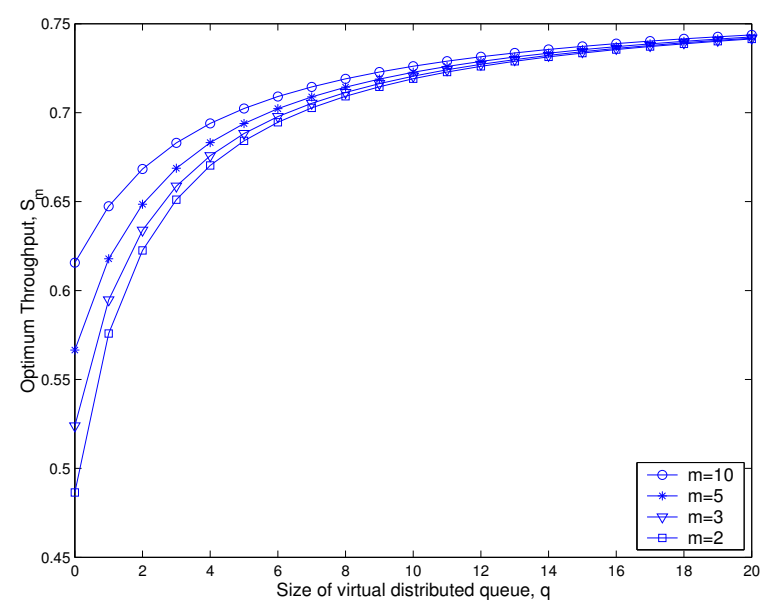

Fig. 6. Optimum throughput of the MAC-mD scheme for $L_{d}=1024$ and for the fixed-total-bandwidth scenario

figure is optimized for $r$, the ratio of the control/data subchannel data rates. A clear improvement of throughput can be observed as $q$ increases, due to the benefits of a larger queue capacity in a queueing system. As one might expect, the performance gain of increasing $q$ is smaller for larger $q$. Therefore, in order to trade-off the complexity of the virtual and distributed queue and the throughput of the corresponding MAC-1D scheme, only a relatively small value of $q$ (e.g., $q=3$ ) is sufficient for the $m=1$ scenario. From Fig. 5, we can also see that the throughput is higher when data packet length is larger. This is due to a lower control packet overhead.

2) Multiple data sub-channels MAC- $m D$ Schemes $(m>1)$ : Figure 6 presents the throughput of the MAC-mD schemes with $m$ data sub-channels as a function of $q$, the size of the virtual and distributed queue. The data packet length is fixed at $L_{d}=1024$. Patterns similar to those in Fig. 5 can be observed, as the throughput increases with $q$. For a fixed $q$, the optimum throughput of the MAC-mD scheme is larger for larger $m$. The difference is more significant when $q$ is small, because the queueing system capacity is the summation of the number of data sub-channels and $q$; i.e., $m+q$. As the queueing system capacity increases, the throughput improves. The performance gain of the throughput due to an increase of $q$ is smaller when $q$ is larger, similarly to the results in Fig. 5. Note that, however, the $r^{*}$ values used to achieve the optimum performance of the MAC-mD schemes are different for different values of $m$.

Figure 7 shows the trend of the maximum achievable throughput of the MAC-mD scheme with the different number of data sub-channels, when the queue size is the same as the number of data sub-channels. It can be seen that the maximum achievable throughput for the MAC-mD scheme increases with an increase in the value of $m$. Overall, this throughput approaches the throughput of the MAC-1 scheme asymptotically with increasing $m$. We show the throughput of each of the two schemes for different data packet length, $L_{d}$. As expected, the throughput performance improves as $L_{d}$ increases.

From Fig. 4, we observe that there is an optimum value of $r$, $r^{*}$, which maximizes the throughput of the MAC-mD scheme.

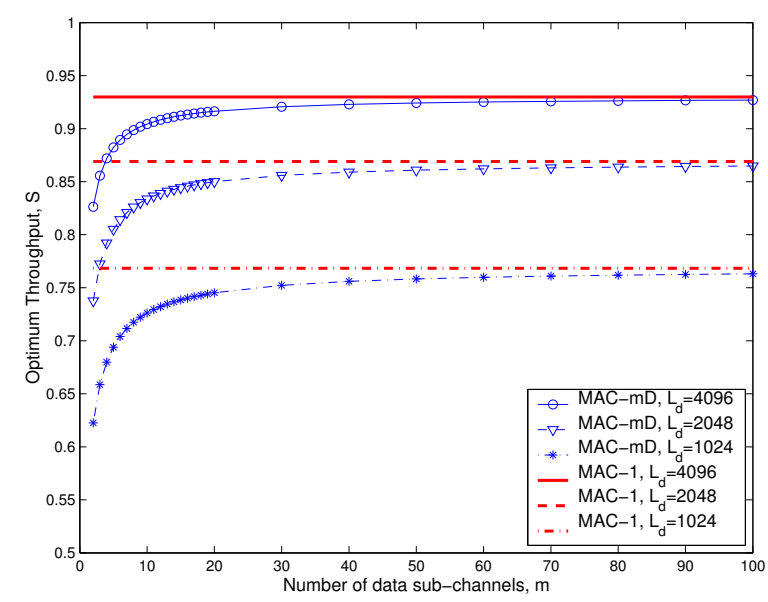

Fig. 7. Throughput of the MAC-mD scheme for $q=m$, for different values of $m$ and $L_{d}$, and for the fixed-total-bandwidth scenario

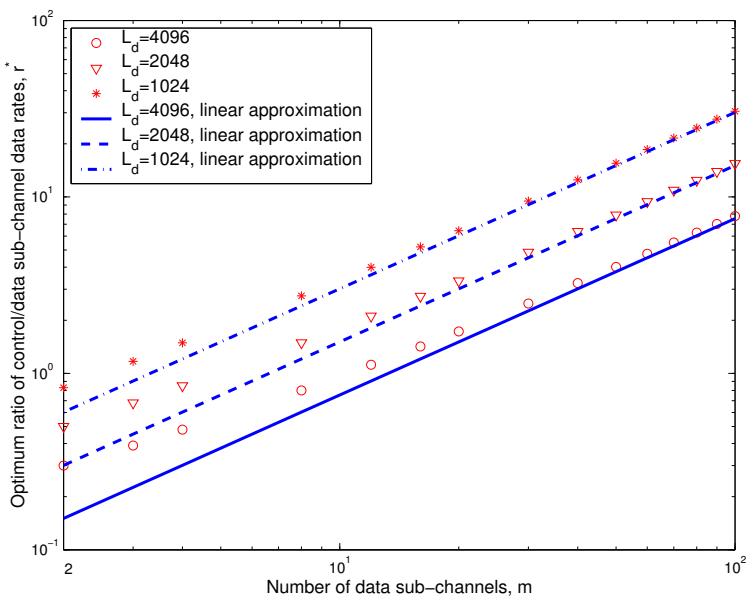

Fig. 8. Optimum $r$ for different values of $m$ and $L_{d}$ and for the fixed-totalbandwidth scenario

We show the relation between $r^{*}$ and $m$ for different $L_{d}$ in Fig. 8. As mentioned in Section V-D, when $m$ is large enough, $\lambda k r^{*} \simeq m$; in Fig. 8, we also plot the lines corresponding to this relation between $r^{*}$ and $m$. As shown in the figure, the linear relation between $r^{*}$ and $m$ can be observed when $m$ is large, but as expected, this linear relation is less accurate when $m$ is small.

In the fixed-channel-bandwidth scenario, such as for CDMA-based channels, $r$ is always 1 because each subchannel has the same data rate. In this case, an optimal number of data sub-channels can be determined as to maximize the throughput of the MAC-mD schemes. Figure 9 shows the throughput of the MAC-mD schemes with $r=1$ and $q=m$. Optimum values of $m$, which maximize the throughput of the MAC-mD schemes, can be observed in the figure. When $m$ is too small, the control sub-channel is idle most of the time, leading to waste of bandwidth and to small throughput. As $m$ increases, the control and data sub-channels match each other. For larger $m$ values, the control sub-channel cannot generate enough successful RTS/CTS dialogues to "fill" these data sub-channels, and so some of data sub-channel remain idle, wasting portion of their capacity. Consequently, the optimum 


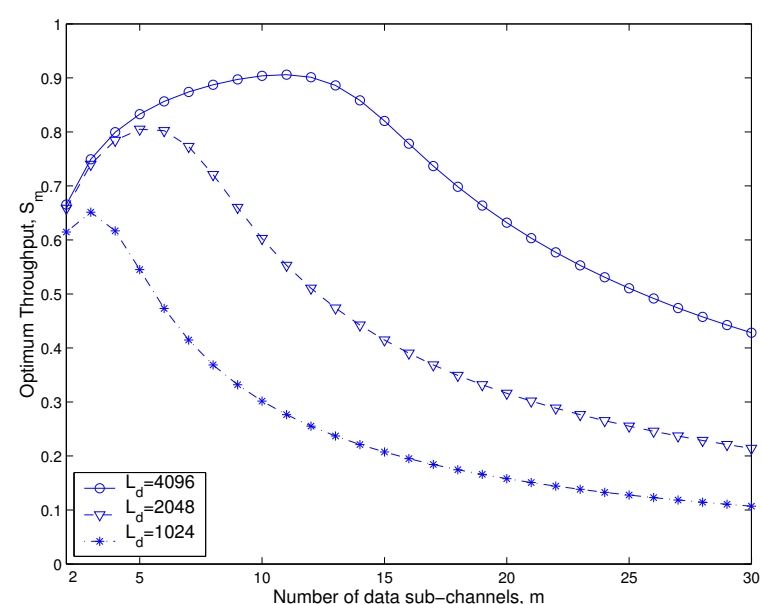

Fig. 9. Throughput of the MAC-mD scheme for $q=m$ and for the fixedchannel-bandwidth scenario $(r=1)$

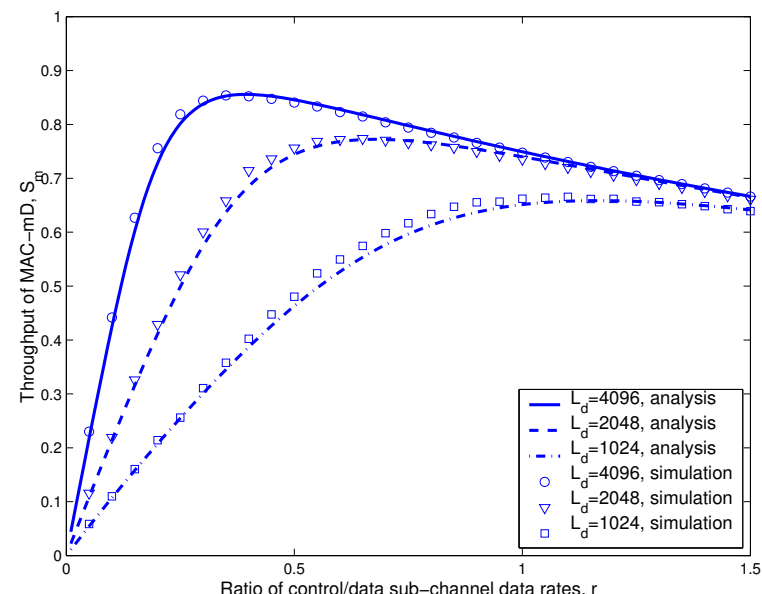

Fig. 10. Simulation and analytical results of the throughput of the MAC-mD schemes for $m=3, q=3$, variable data-packet length, and for the fixedtotal-bandwidth scenario

value of $m$ is larger for larger values of $L_{d}$.

\section{B. Simulation Results}

We used a packet-level simulation program, which we wrote in $\mathrm{C}++$, to study the performance of our analytical models. ${ }^{7}$ The simulated network was a fully-connected network with $N=50$ nodes and with different values of $L_{d}$ and $m$ $(q=m)$. Data packet lengths were either fixed or variable with exponential distribution. The control-packet length was assumed to be 48 bits. The channel data rate was $1 \mathrm{Mbps}$. The simulations ran for 10 seconds, simulation time. The operation of the MAC-mD schemes was as presented in Section IV. Data packets arrived at each of the $N$ nodes according to the Poisson distribution. Note that nodes are assumed to know the precise states of the distributed queue. Therefore, we simulate the MAC schemes with a perfect distributed queue.

In Fig. 10, we compare the simulation results with analytical results as a function of $r$, for networks with variable and

\footnotetext{
${ }^{7} \mathrm{We}$ did not use other simulators such as OPNET or NS-2 because their MAC models include other features, e.g., back-off and slotted operation, that are not presented in our analytical model.
}

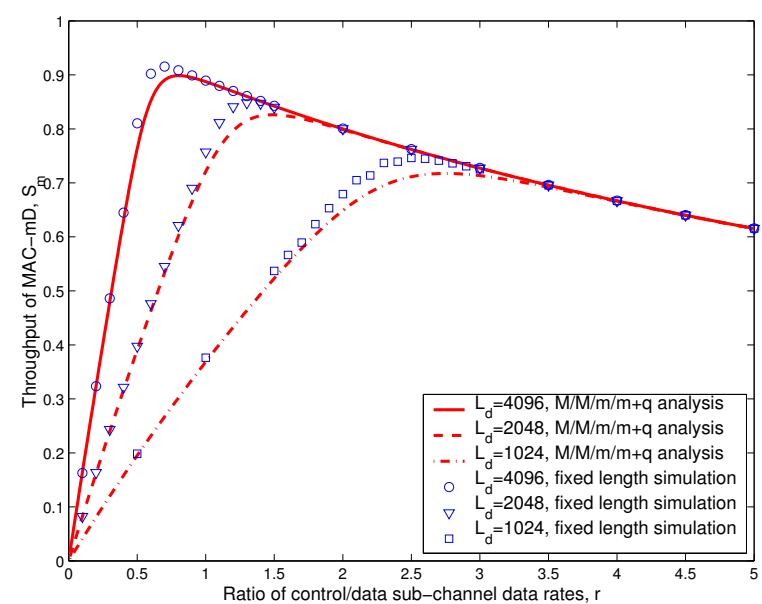

Fig. 11. Simulation and analytical results of the throughput of the MAC-mD schemes for $m=8, q=8$, different values of $L_{d}$, and for the fixed-totalbandwidth scenario

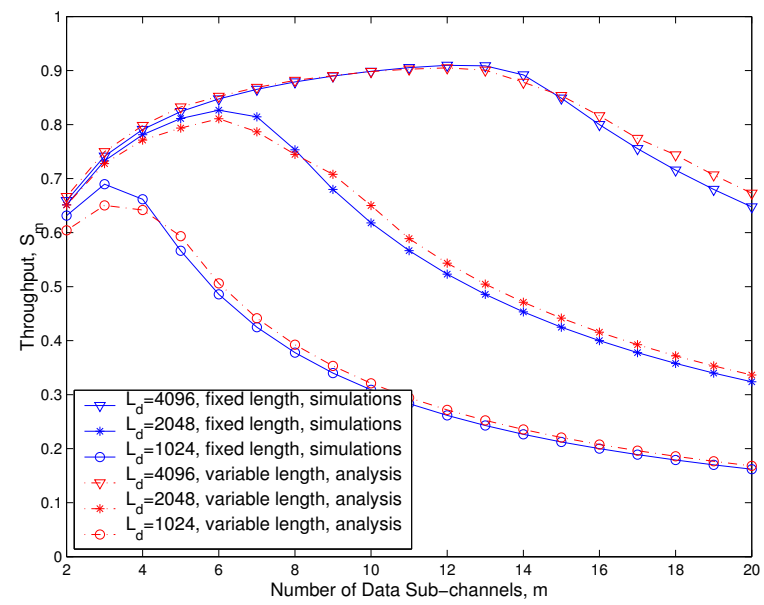

Fig. 12. Simulation and analytical results of the throughput of the MAC-mD scheme for different values of $m$ and $L_{d}$, and for the fixed-channel-bandwidth scenario

exponentially distributed data-packet length. The value of $m$ and $q$ are both assumed to be 3 in this figure. The expected length of data packets for these simulations was set to 1024, 2048, and 4096. As evident from the figure, the simulation results agree quite well with the analytical results, strongly suggesting the validity of our analytical model.

Since our analysis does not study the throughput performance of the MAC-mD scheme with fixed data packet length for $m>1$, we compared the difference in performance of the MAC-mD schemes with fixed data-packet length and with variable data-packet length. These results are depicted in Fig. 11, for $m=8$ and $q=8$. Additional results of this comparison for other values of $m$ are shown in Figure 12, where the data packet length is fixed for three of the curves and exponentially distributed for the other three curves. The close match between the results for fixed and for variable datapacket length indicating that our $\mathrm{M} / \mathrm{M} / \mathrm{m} / \mathrm{m}+\mathrm{q}$ analytical model is sufficient to reasonably well approximate the performance of the MAC-mD schemes with fixed data packet length, especially when $m$ is large. 


\section{Summary, CONClusions, AND Future WORK}

In a wireless communication network, the Medium Access Control (MAC) scheme can significantly affect the network's performance. In particular, due to the stochastic traffic patterns and due to the fact that the communication medium is shared among a multitude of network nodes, an efficient MAC scheme is essential for adequate performance of the communication process. To improve the throughput performance of MAC schemes for random-accessed medium, especially in high data rate systems, some researchers proposed to use multiple subchannels instead of a single shared channel. However, there has been no comprehensive performance comparison study to indicate under what conditions the multiple-channel scheme is preferable over the single-channel scheme.

In this paper, we analyzed a family of generic multiplechannel MAC schemes (MAC-mD), which use one subchannel, the control sub-channel, for transmission of the RTS/CTS dialogues, while the other sub-channels, the data sub-channels, are assigned to transmissions of the actual data packets. The RTS packets contend on the right to use one of the data sub-channels and the winner in a contention is reserved the right to use one of the data sub-channels to transmit, collision free, its data packet.

Based on the pure ALOHA contention-resolution technique on the control sub-channel, we have developed a queueing model to study the performance of the MAC-mD schemes for the fixed-total-bandwidth scenario and for the fixed-channelbandwidth scenario. In the fixed-total-bandwidth scenario, the total bandwidth, hence, the maximum data rate, is fixed. The larger the number of sub-channels is, the smaller is the data rate of each sub-channel. Our analysis shows that such multiple-channel MAC schemes are always out-performed by the corresponding single shared channel schemes, given that the propagation delays are negligible. In the fixed-channelbandwidth scenario, each sub-channel has the same data rate. Our study shows that there is an optimum number of subchannels in such MAC schemes, as to maximize the total network throughput. This optimum number can be calculated numerically based on our analytical model. Our numerical results show that the optimum number of sub-channels depends largely on the ratio of the data packet length to the control packet length.

The analytical framework proposed in this paper can be used to derive the optimum ratio of data rate of control sub-channel to that of the data sub-channel in the fixed-total-bandwidth scenario. In the fixed-channel-bandwidth scenario, our model can be used to calculate the optimum number of data subchannels based on the ratio of data packet length to control packet length.

The significance of the results of our work and the message of this paper to the technical community is that, while using multiple sub-channels instead of a single shared channel may solve certain problems, such as reducing or even eliminating collisions between data packets and control packets, overall it may not improve the throughput performance of a MAC scheme. Our study shows that a single-channel MAC scheme always out-performs the corresponding multi-channel MAC schemes in the cases considered; i.e., negligible propagation delay and fully-connected network topology.

Even though our results are derived for MAC protocols that are based on the RTS/CTS dialogue and on the pure ALOHA contention resolution technique, these results are also applicable to other related multiple-channel MAC schemes. For instance, the well-known IEEE 802.11 standard [16] uses the CSMA/CA as its access scheme. The CSMA/CA scheme is essentially similar to the MAC-1 scheme studied in [13], but with additional mechanisms, such as carrier sensing and backoff control. These additional mechanisms improve the rate of successful RTS/CTS dialogues. Nevertheless, our results and conclusions regarding to the comparison of single-channel and multiple-channel MAC schemes still apply.

In our future work, we will extend our model to multiple control sub-channels. When multiple transceivers are available in a single network node, it is possible to pipeline the contention windows and the data transmissions on different subchannels. It would be interesting to study the performance of multi-channel MAC schemes in such network environments. Furthermore, it would be of particular interest to study the performance of the multi-channel MAC schemes in multihop wireless networks, which allow spatial reuse of data subchannels.

\section{REFERENCES}

[1] R. G. Gallager, "A perspective on multiaccess channels," IEEE Trans. on Information Theory, vol. IT-31, no. 2, pp. 124-142, March 1985.

[2] P. Karn, "MACA - a new channel access method for packet radio," in ARRL/CRRL Amateur Radio 9th Computer Networking Conference, 1990, pp. 134-140.

[3] F. A. Tobagi and L. Kleinrock, "Packet switching in radio channels: Part III-polling and (dynamic) split-channel reservation multiple access," IEEE Trans. on Communications, vol. COM-24, no. 8, pp. 832-845, August 1976.

[4] D. H. C. Du, S.-P. Chang, and G. Subbaro, "Multiple packet multiple channel CSMA/CD protocols for local area networks," in Proc. of the 7th Int. Annual Joint Conf. of the IEEE Computer and Communications Societies (INFOCOM '89), April 1989.

[5] W.-C. Hung, K. L. E. Law, and A. Leon-Garcia, "A dynamic multichannel MAC for Ad Hoc LAN," in Proc. 21st Biennial Symposium on Communications, June 2-5 2002, pp. 31-35, Kingston, Canada.

[6] A. Tzamaloukas and J.J. Garcia-Luna-Aceves, "A receiver-initiated collision-avoidance protocol for multi-channel networks," in Proc. of the 19th Int. Annual Joint Conf. of the IEEE Computer and Communications Societies (INFOCOM '01), April 2001.

[7] J. So and N. H. Vaidya, "Multi-channel MAC for ad hoc networks: Handling multi-channel hidden terminals using a single transceiver," in Proc. of ACM International Symposium on Mobile Ad Hoc Networking and Computing (MobiHoc '04), Roppongi Hills, Tokyo, Japan, May 24262004.

[8] S.-L. Wu, Y.-C. Tseng, C.-Y. Lin, and J.-P. Sheu, "A multi-channel MAC protocol with power control for multi-hop mobile ad hoc networks," Computer Journal, vol. 45, no. 1, pp. 101-110, 2002.

[9] A. R. Raghavan and C. W. Baum, "An unslotted multichannel channelaccess protocol for distributed direct-sequence networks," Mobile Networks and Applications, vol. 5, no. 1, pp. 49-56, March 2000.

[10] S. Singh and C. S. Raghavendra, "PAMAS - power aware multiaccess protocol with signaling for ad hoc networks," ACM Computer Communications Review, vol. 28, no. 3, pp. 5-26, July 1998.

[11] X. Yang, N. H. Vaidya, and P. Ravichandran, "Split-channel pipelined packet scheduling for wireless networks," IEEE Trans. on Mobile Computing, 2004, in press.

[12] A. Muqattash and M. Krunz, "Power controlled dual channel (PCDC) medium access protocol for wireless ad hoc networks," in Proc. of the 21 st Int. Annual Joint Conf. of the IEEE Computer and Communications Societies (INFOCOM '03), April 2003. 
[13] J. Deng, Y. S. Han, and Z. J. Haas, "Analyzing split channel medium access control schemes," IEEE Trans. on Communications, 2005, in press.

[14] N. Abramson, "The ALOHA system - another alternative for computer communications," in AFIPS Conf. Proc. of Fall Joint Computer Conference, 1970, vol. 37, pp. 281-285.

[15] T. D. Todd and J. W. Mark, "Capacity allocation in multiple access networks," IEEE Trans. on Communications, vol. COM-33, no. 11, pp. 1224-1226, November 1985.

[16] IEEE 802.11, "Wireless LAN MAC and physical layer specifications," June 1999.

[17] H. Takagi, Queueing Analysis, North-Holland, Amsterdam, Netherlands, 1993.

[18] S. K. Bose, An Introduction to Queueing Systems, Kluwer Academic/Plenum Publishers, New York, NY, 2002.

[19] D. Bertsekas and R. Gallager, Data Networks, Prentice-Hall, Upper Saddle River, NJ, USA, 2nd edition, 1992.

\section{APPENDIX}

\section{AN IMPLEMENTATION OF THE Distributed QUEUE}

It is actually rather difficult and inefficient to implement a distributed queue to capture the states perfectly. Therefore, the performance of the MAC-mD schemes that we investigate in this work represents the performance upper bound of real MAC schemes in this category.

Our implementation is based on the assumption of fixed packet length. An implementation that works with variable packet-length is also possible but more complicated (note that in such cases, the RTS/CTS packets may carry the packet length information), but due to page limitations, we will not discuss it here.

Assume that the $m$ data sub-channels can be identified from 0 to $m-1$. Each node maintains three counters: $s$-counter to record the number of customers in service and in queue; $p$-counter to record the position of local node in the queue; and $d$-counter to record the ID of the next available data subchannel. Initially, all counters are set to zero.

When a successful RTC/CTS exchange takes place, the sender and the receiver check the $s$-counter. If $s<m$, they pick the data sub-channel as indicated by the $d$-counter. The $s$-counter is incremented on all nodes. Furthermore, the $d$ counter is then incremented $(\bmod m)$ on all nodes. If $m \leq s<$ $m+q$, the sender and the receiver record the $s-m$ value into their $p$-counters, where $s$ is the content of the $s$-counter. The $s$-counter is then incremented on all nodes. If $s \geq m+q$, the sender and the receiver should not join the distributed queue, but return to compete on the control sub-channel. ${ }^{8}$

Through time-out events or through monitoring of the data sub-channel, nodes notice when the transmission of a data packet is completed on a data sub-channel. Then the $s$-counter and the $p$-counter are decremented on all nodes. When the $p$ counter becomes zero, the sender and the receiver should use the $d$-th data sub-channel for data transmission. The $d$-counter is further incremented $(\bmod m)$ on all the nodes.

\footnotetext{
${ }^{8} \mathrm{~A}$ more practical approach is to stop competing on the control subchannel when $s \geq m+q$. However, such an approach may reduce the overall throughput slightly because the control sub-channel is left idle before $s$ becomes smaller than $m+q$.
}

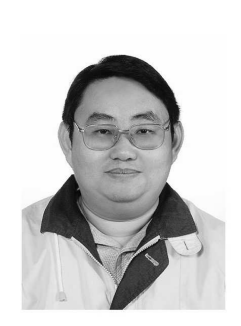

Yunghsiang S. Han (S'90-M'93) was born in Taipei, Taiwan, on April 24, 1962. He received the B. S. and M. S. degrees in electrical engineering from the National Tsing Hua University, Hsinchu, Taiwan, in 1984 and 1986, respectively, and the Ph. D. degree from the School of Computer and Information Science, Syracuse University, Syracuse, $\mathrm{NY}$, in 1993. He is a winner of 1994 Syracuse University Doctoral Prize. From 1993 to 1997 he was an Associate Professor in the Department of Electronic Engineering at Hua Fan College of Humanities and Technology, Taipei Hsien, Taiwan. From 1997 to 2004 he was with the Department of Computer Science and Information Engineering at National Chi Nan University, Nantou, Taiwan. He was promoted to Full Professor in 1998. From June to October 2001 he was a visiting scholar in the Department of Electrical Engineering at University of Hawaii at Manoa, HI, and from September 2002 to January 2004 he was the SUPRIA visiting research scholar in the Department of Electrical Engineering and Computer Science and CASE center at Syracuse University, NY. He is now with the Graduate Institute of Communication Engineering at National Taipei University, Taipei, Taiwan. His research interests are in wireless networks, security, and error-control coding.

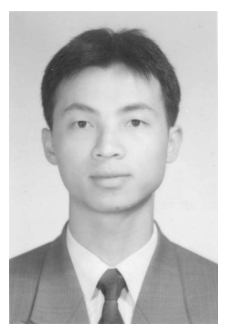

Jing Deng (S'98-M'02) received the B.E. and M.E. degrees in Electronic Engineering from Tsinghua University, Beijing, P. R. China, in 1994 and 1997, respectively, and the Ph.D. degree in Electrical and Computer Engineering from Cornell University, Ithaca, NY, in 2002.

Dr. Deng is an assistant professor in the Department of Computer Science at the University of New Orleans. From 2002 to 2004, he visited the CASE center and the Department of Electrical Engineering and Computer Science at Syracuse University, Syracuse, NY as a research assistant professor, supported by the Syracuse University Prototypical Research in Information Assurance (SUPRIA) program. He was a teaching assistant from 1998 to 1999 and a research assistant from 1999 to 2002 in the School of Electrical and Computer Engineering at Cornell University. His interests include mobile ad hoc networks, wireless sensor networks, wireless network security, energy efficient wireless networks, and information assurance.

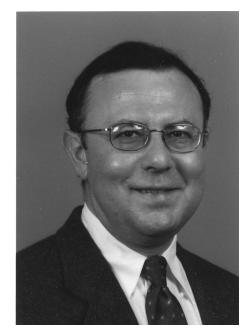

Zygmunt J. Haas (S'84-M'88-SM'90) received his B.Sc. in EE in 1979 and M.Sc. in EE in 1985. In 1988, after earning his Ph.D. from Stanford University, he joined the AT\&T Bell Laboratories in the Network Research Department. There he pursued research on wireless communications, mobility management, fast protocols, optical networks, and optical switching. From September 1994 till July 1995, Dr. Haas worked for the AT\&T Wireless Center of Excellence, where he investigated various aspects of wireless and mobile network technologies. In August 1995, he joined the faculty of the School of Electrical and Computer Engineering at Cornell University, where he is now a Professor and the Associate Director for Academic Affairs.

Dr. Haas is an author of numerous technical conference and journal papers and holds fifteen patents in the areas of high-speed networking, wireless networks, and optical switching. He has organized several workshops, delivered numerous tutorials at major IEEE and ACM conferences, and serves as editor of several journals and magazines, including the IEEE Transactions on Networking, the IEEE Transactions on Wireless Communications, the IEEE Communications Magazine, and the ACM/Kluwer Wireless Networks journal. He has been a guest editor of IEEE JSAC issues on "Gigabit Networks," "Mobile Computing Networks," and "Ad-Hoc Networks." Dr. Haas served as a Chair of the IEEE Technical Committee on Personal Communications and is currently serving as the Chair of the Steering Committee of the IEEE Pervasive Computing magazine. His interests include: mobile and wireless communication and networks, performance evaluation of large and complex systems, and biologically inspired networks. His e-mail is: haas@ece.cornell.edu and his URL is: http://wnl.ece.cornell.edu . 